\title{
WNT signaling determines tumorigenicity and function of ESC-derived retinal progenitors
}

\author{
Lu Cui,, 1,2,3 Yuan Guan, ${ }^{1,2}$ Zepeng Qu,1,3 Jingfa Zhang, ${ }^{2}$ Bing Liao,,1,3 Bo Ma, ${ }^{4}$ Jiang Qian, ${ }^{4}$ \\ Dangsheng Li, ${ }^{5}$ Weiye $\mathrm{Li},{ }^{6}$ Guo-Tong $\mathrm{Xu},{ }^{2}$ and Ying Jin ${ }^{1,3}$
}

\begin{abstract}
${ }^{1}$ Key Laboratory of Stem Cell Biology, Institute of Health Sciences, Shanghai Jiao Tong University School of Medicine, and Shanghai Institutes for Biological Sciences, Chinese Academy of Sciences, Shanghai, People's Republic of China. ${ }^{2}$ Department of Ophthalmology, Shanghai Tenth People's Hospital and Tongji Eye Institute, Tongji University School of Medicine, Shanghai, People's Republic of China. 3Department of Molecular Developmental Biology, Shanghai Jiao Tong University School of Medicine, Shanghai, People's Republic of China. ${ }^{4}$ Department of Ophthalmology, Eye, Ear, Nose, Throat Hospital, Fudan University, Shanghai, People's Republic of China. ${ }^{5}$ Shanghai Institutes for Biological Sciences, Chinese Academy of Sciences, Shanghai, People's Republic of China. ${ }^{6}$ Department of Ophthalmology, Drexel University College of Medicine, Media, Pennsylvania, USA.
\end{abstract}

\begin{abstract}
Tumor formation constitutes a major obstacle to the clinical application of embryonic stem cell-derived (ESC-derived) cells. In an attempt to find major extracellular signaling and intrinsic factors controlling tumorigenicity and therapeutic functionality of transplanted ESC-derived retinal progenitor cells (ESCRPCs), we evaluated multiple kinds of ESC-RPCs in a mouse retinal degeneration model and conducted genome-wide gene expression profiling. We identified canonical WNT signaling as a critical determinant for the tumorigenicity and therapeutic function of ESC-RPCs. The function of WNT signaling is primarily mediated by TCF7, which directly induces expression of Sox 2 and Nestin. Inhibition of WNT signaling, overexpression of dominant-negative $T c f 7$, and silencing $T c f 7$, Sox2, or Nestin all resulted in drastically reduced tumor formation and substantially improved retinal integration and visual preservation in mice. These results demonstrate that the WNT signaling cascade plays a critical role in modulating the tumorigenicity and functionality of ESC-derived progenitors.
\end{abstract}

\section{Introduction}

The unique properties of embryonic stem cells (ESCs), unlimited self-renewal and pluripotency, make them an attractive cell source for the treatment of various degenerative diseases. However, the same properties also present a major hurdle for their clinical application. Tumor formation has been reported in the transplantation of ESC derivatives despite predifferentiation or presorting (1-6), raising a safety concern for the therapeutic use of ESC-derived cell products in humans. On the other hand, transplantation of photoreceptor precursors in neonatal mice repaired retinal defect efficiently without development of any tumors $(7,8)$, which emphasizes the critical role of the developmental stage of donor cells in determining the cell fate following transplantation. Thus, steering ESCs to an appropriate state could be an important step for safe and effective cell therapies.

To date, ESCs from the mouse, monkey, and human have been successfully differentiated into retinal cells in vitro (9-13). Sasai's group developed an efficient induction of retinal precursors by culturing mouse ESCs under a serum-free suspension condition (SFEB culture) and obtained high percentages of differentiated cells expressing key eye-field transcription factors (12-14). However, mechanisms governing efficient generation of various types of retinal cells and the optical cups from ESCs in vitro as well as their application potential in vivo, in regards to the functional integration and safety, are not clearly elucidated.

In an attempt to find major extracellular signaling and intrinsic factors controlling tumorigenicity and therapeutic effects of ocular transplanted ESC-derived retinal progenitor cells (ESC-RPCs),

Authorship note: Lu Cui and Yuan Guan contributed equally to this work. Conflict of interest: The authors have declared that no conflict of interest exists. Citation for this article: J Clin Invest. 2013;123(4):1647-1661. doi:10.1172/JCI65048. we identified the canonical WNT signaling-activated TCF7-SOX2NESTIN cascade as a critical determinant for the consequence of ESC-RPC transplantation: whether tumors would form as well as whether successful integration into the host retina and prevention of the visual defect would be achieved. Canonical WNT signaling is known to play a key role in cell fate determination for various cell lineages, and its inappropriate activation is frequently associated with cancers (15-17). It has also been shown to promote proliferation of isolated retinal stem cells (18) and retina regeneration in adult mammals (19). However, the correlation between WNT signaling in ESC-derived donor cells and their therapeutic effect as well as tumorigenicity after transplantation in a disease model remains unexamined. In addition, factor(s) that mediate the function of WNT signaling in the control of cell fate commitment remain elusive. In this study, we link the activity of WNT signaling to the tumorigenic potential of ESC-RPCs and provide the experimental evidence for transcriptional factor TCF7 to regulate expression of SOX2 and NESTIN, two important genes actively engaged in the neural development and tumor formation. The tumorigenic and therapeutic effect of transplanted ESC-RPCs is determined in a well-studied sodium iodate-induced (SI-induced) mouse retinal degeneration model $(20,21)$. We also show that the expression of TCF7, SOX2, and NESTIN is closely associated in mouse neonatal retinae. These findings open new avenues to define and manipulate ESC-derived donor cells prior to transplantation for safe and effective cell therapies.

\section{Results}

ESC-RPCs but not primary retinal progenitor cells produce neural tumors in transplanted eyes. We began with differentiation of mouse ESCs (46C line containing a Sox1.EGFP-targeted gene) $(22,23)$ into retinal progenitor cells (RPCs) using a chemically defined SFE- 


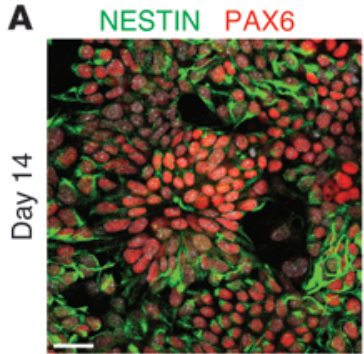

PAX6 OTX2
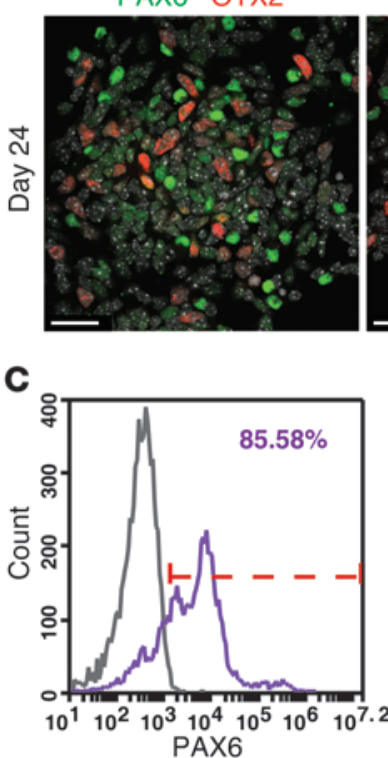

D

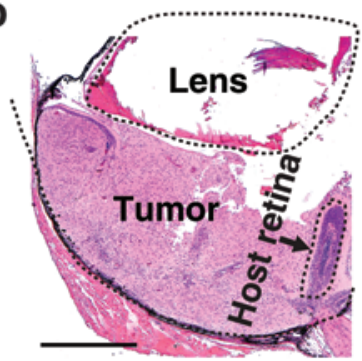

PAX6 SIX3



PAX6 NRL



PAX6 RAX

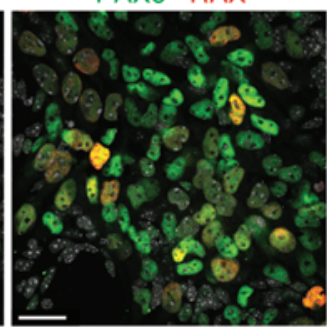

OPSIN

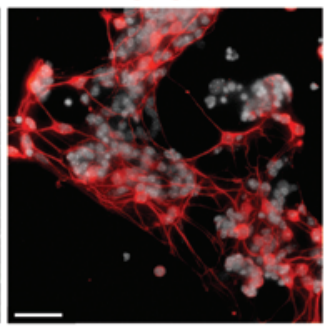

B
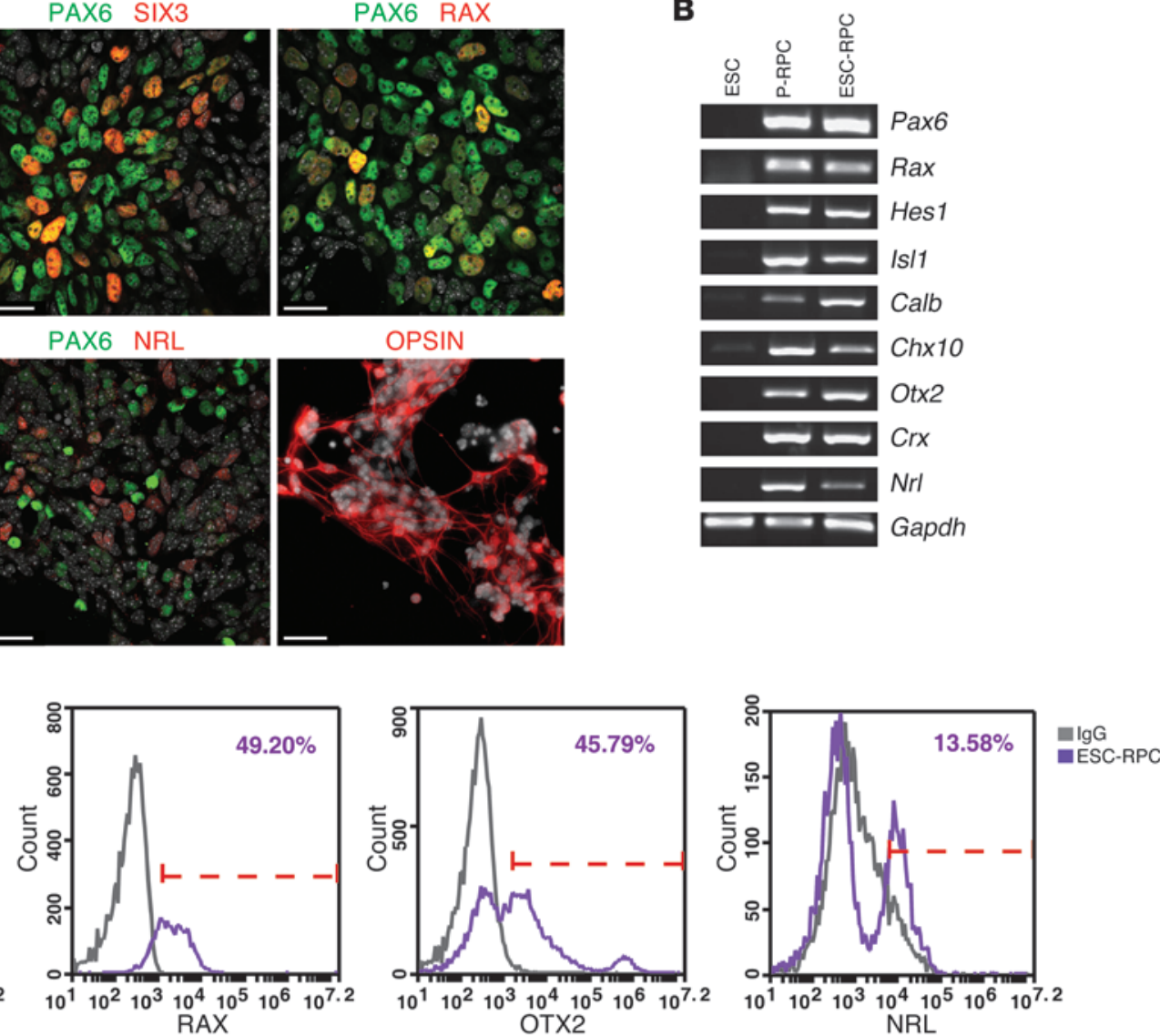

E

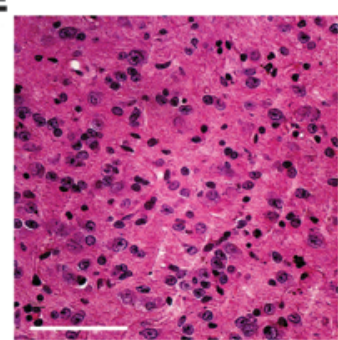

F


G
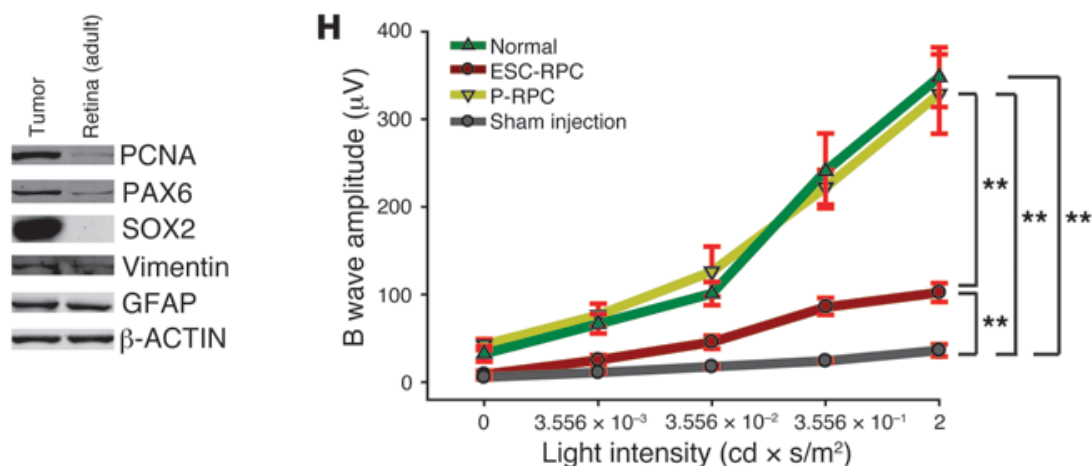


\section{Figure 1}

ESC-RPC but not P-RPC transplantation generates ocular tumors. (A) Immunofluorescence images of costaining of PAX6 with NESTIN, SIX3, and RAX, respectively, in ESC-RPCs at 14 days and costaining of PAX6 with OTX2 and NRL, respectively, or opsin staining alone at 24 days of the differentiation. DAPI is shown in gray. (B) Representative RT-PCR analysis of the expression for retinal markers in ESCs, P-RPCs, and ESC-RPCs. (C) FCM profiles of ESC-RPCs for subpopulations expressing PAX6, RAX, OTX2, and NRL, respectively. (D) H\&E staining of the section of an ocular tumor 3 weeks after ESC-RPC injection. (E) The magnified image of tumor tissue in $\mathbf{D}$. (F) A cryosection of a transplanted eyeball containing the tumor tissue from injected ESC-RPCsEGFP (green). (G) Protein levels of several markers in tumor tissues were compared with those in the normal adult retina. $(\mathbf{H})$ The amplitude of ERG b waves at different light intensities is shown for normal mice and SI-treated mice after subretinal injection of P-RPCs, ESC-RPCs, and the cell culture medium (Sham injection), respectively. Mean \pm SD; ANOVA; ${ }^{* \star} P<0.01 ; n=10$ for each group. Scale bar: $25 \mu \mathrm{m}(\mathbf{A}) ; 500 \mu \mathrm{m}$ (D); $100 \mu \mathrm{m}$ (E and F).

B-based protocol modified from a previously reported SFEB-based approach (ref. 12, Supplemental Figure 1, A-C, and Supplemental Methods; supplemental material available online with this article; doi:10.1172/JCI65048DS1). According to this protocol, ESCRPCs were obtained by selection of SOX1.EGFP+ neural progenitors through FACS at day 7 of differentiation, followed by further differentiation into RPCs until day 24. Differentiated cells were characterized by 3 independent approaches. Immunofluorescence staining showed that they expressed multiple neural progenitor and retinal markers, such as NESTIN, PAX6, SIX3, and RAX for RPCs at an early stage of differentiation (day 14), OTX2 and NRL for photoreceptor precursors, and Rhodopsin (OPSIN) for rod photoreceptors at the end of differentiation (day 24) (Figure 1A). Hereafter, ESC-derived RPCs at day 24 were termed ESC-RPCs. Then, transcript levels of various markers among ESCs, ESC-RPCs, and primary RPCs (P-RPCs) isolated from neonatal mice at P1 were compared. Results of RT-PCR assays revealed that pluripotency-associated genes (Oct4, Nanog, and Rex1) were not detectable in ESC-RPCs and P-RPCs (data not show), whereas markers for various types of retinal cells were found in both ESC-RPCs and P-RPCs (Figure 1B; see Supplemental Table 1 for primers). Western blot analysis confirmed the absence of OCT4 and NANOG proteins in ESC-RPCs and P-RPCs (Supplemental Figure 1D). Moreover, the component of retinal cell types in ESC-RPCs was characterized by flow cytometry (FCM) analysis. The majority of ESC-RPCs were PAX6 ${ }^{+}$. A fraction of ESC-RPCs was $\mathrm{RAX}^{+}$or $\mathrm{OTX}^{+}$, and $13.58 \%$ of cells expressed NRL (Figure $1 \mathrm{C}$ and Supplemental Figure 1E). Thus, ESC-RPCs were composed of a mixture of neural retinal progenitors and committed photoreceptors.

We then tested the therapeutic potential and risk of tumor formation by injecting $8 \times 10^{4}$ of ESC-RPCs into the subretinal space of SI-treated adult mice. All transplanted ESC-RPCs (except for D3 ${ }^{\text {EGFP-de- }}$ rived RPCs) in this study were labeled by EGFP (ESC-RPCs ${ }^{\text {EGFP) }}$ through infection with a lentiviral vector containing an EGFP sequence at day 24 of differentiation and were injected into mice 4 days later (day 28). The same number of P-RPCs carrying an EGFP transgene (P-RPCs ${ }^{\mathrm{EGFP}}$ ) (24) was used as a transplantation control. Three weeks later, tumors were detected in 63 eyes out of 104 ESC-RPC transplanted eyes, which maintained an intact external structure with the enlarged size (Supplemental Figure 1F). Most internal structures were entirely destroyed (Figure 1D). The cells within tumors seemed to have a homogenous morphology, without any distinct tissue structure (Figure 1E), and were EGFP positive (Figure 1F), indicating the origin of exogenous injection. The expression of SOX2, NESTIN, and PAX6 as well as PCNA was detected in tumor cells, hinting at the existence of immature RPCs (Figure 1G and Supplemental Figure $1 \mathrm{G})$. Furthermore, FACS-isolated $\mathrm{EGFP}^{+}$tumor cells had the capacity to form neurospheres and a high BrdU incorporation rate (Supplemental Figure 1, H and I). Immunostaining data showed that isolated tumor-derived cells were SOX $1^{+}, \mathrm{NESTIN}^{+}$, and $\mathrm{SOX}^{+}$(Supplemental Figure 1J), implicating the neural progenitor nature of tumors. Nevertheless, about 33\% of injected ESCRPCs integrated well without forming tumors. In these eyes, the visual function was partially preserved (see below). This finding argues for the potential of ESC-RPCs to integrate and function in the host retina should tumor formation be prevented. In contrast to ESC-RPCs, strikingly, none of the eyes injected with P-RPCs developed tumors and efficient integration took place in 72 eyes out of 80 transplanted eyes (Table 1).

The visual function of eyes receiving P-RPCs and ESC-RPCs (for tumor-free eyes) was assessed by electroretinography (ERG) analysis, which reflects functional responses of photoreceptors and downstream neurons upon light stimulus (Figure $1 \mathrm{H}$ and Supplemental Figure $1 \mathrm{~K}$ ). In line with previous reports, SI treatment led to a complete loss of ERG response (the sham group; $n=10$ ), as evidenced by the disappearance of scotopic a and b waves, and of oscillatory potentials, the latter representing the blood circulation in the retina and synaptic circuits in the inner nuclear layer. P-RPCs corrected both defects completely $(n=10)$. These results suggest that satisfactory cell transplantation would be achieved if we could steer ESC-RPCs into a state similar to that of P-RPCs.

Inbibition of canonical WNT signaling enables ESC-RPCs to have $P$-RPC-like properties. The distinct results obtained from the transplantation of ESC-RPCs and P-RPCs prompted us to search for molecular networks distinguishing these 2 types of donor cells. To this end, genome-wide transcript profiles were compared between ESC-RPCs and P-RPCs, with 3 replicates for each cell type. A total

\section{Table 1}

Summary of transplantation results of 8 kinds of donor cells used in this study

\begin{tabular}{lcccccc} 
Donor cells & \multicolumn{2}{c}{ Tumor } & \multicolumn{2}{c}{ Integration } & \multicolumn{2}{c}{ Failure } \\
& No. & $\%$ & No. & $\%$ & No. & $\%$ \\
P-RPC & - & - & $72 / 80$ & $90 \%$ & $8 / 80$ & $10 \%$ \\
ESC-RPC & $63 / 104$ & $60.58 \%$ & $35 / 104$ & $33.65 \%$ & $6 / 104$ & $5.77 \%$ \\
DKK1-ESC-RPC & $2 / 62$ & $3.23 \%$ & $58 / 62$ & $93.54 \%$ & $2 / 62$ & $3.23 \%$ \\
ANTcf7-ESC-RPC & $6 / 132$ & $4.55 \%$ & $102 / 132$ & $77.27 \%$ & $24 / 132$ & $18.18 \%$ \\
ShSox2-ESC-RPC & $6 / 122$ & $4.92 \%$ & $79 / 122$ & $64.75 \%$ & $37 / 122$ & $30.33 \%$ \\
shNestin-ESC-RPC & $12 / 92$ & $13.04 \%$ & $65 / 92$ & $70.65 \%$ & $15 / 92$ & $16.31 \%$ \\
shTcf7-ESC-RPC & $2 / 36$ & $5.56 \%$ & $25 / 36$ & $69.44 \%$ & $9 / 36$ & $25 \%$ \\
shControl-ESC-RPC & $45 / 60$ & $75 \%$ & $12 / 60$ & $20 \%$ & $3 / 60$ & $5 \%$ \\
& & & & & &
\end{tabular}

All ESC-RPCs listed in this table were derived from the $46 \mathrm{C}$ cell line. No tumors were found in P-RPCs. Failure is defined by the absence of EGFP+ donor cells in injected eyes. 
A

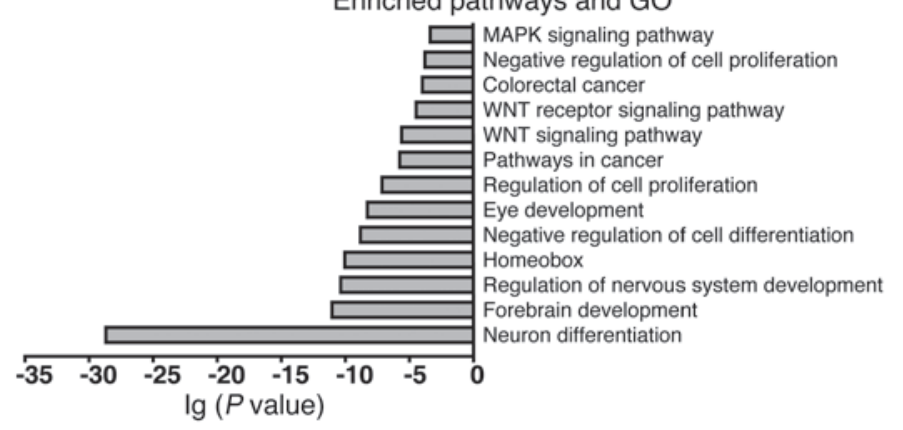

D



B WNT signaling pathway

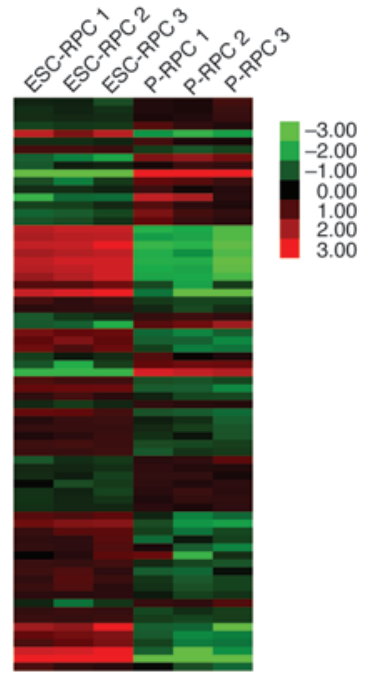

E

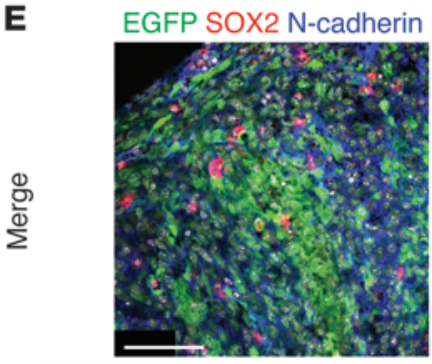

EGFP GFAP TUJ1
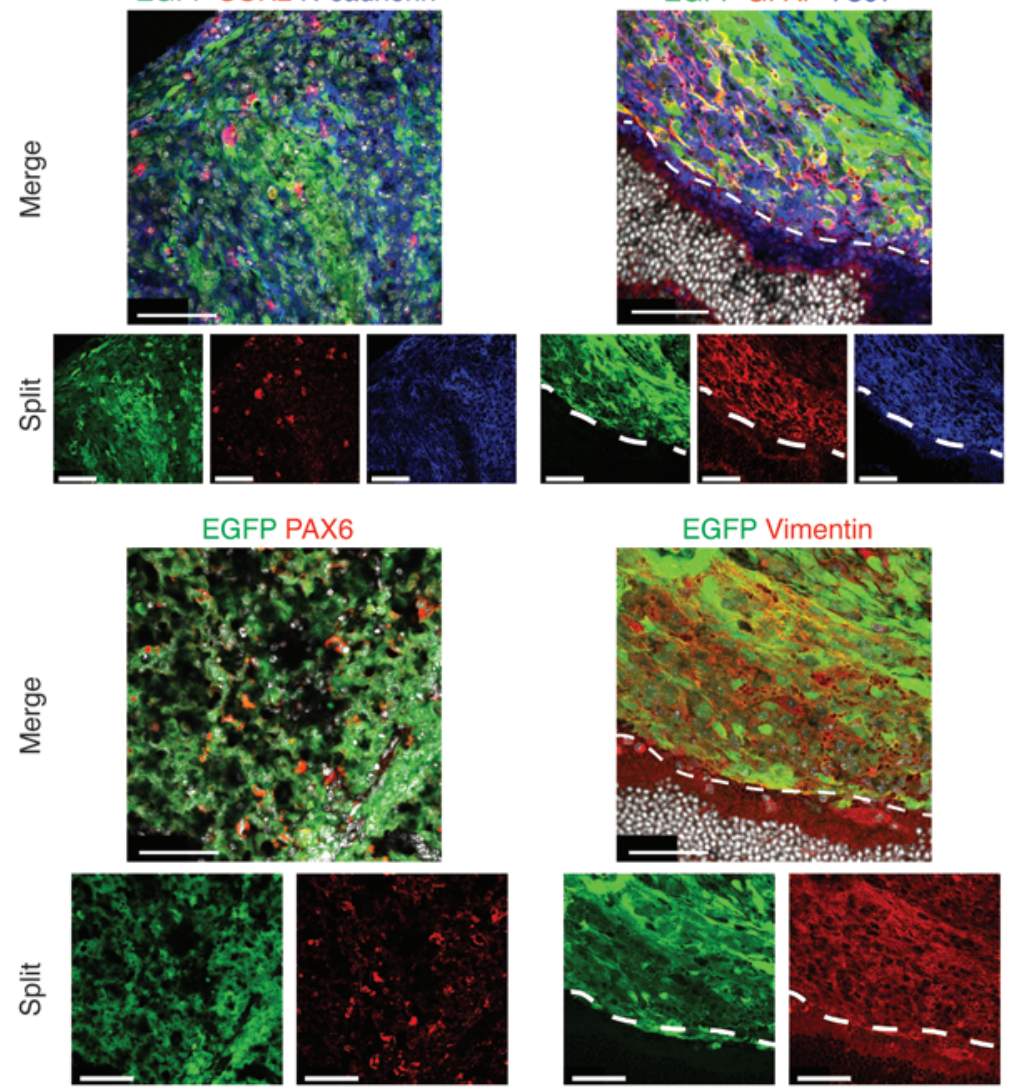

EGFP Vimentin


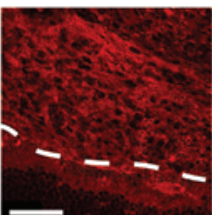



EGFP fTCF1 NESTIN

EGFP $\beta$-Catenin 


\section{Figure 2}

WNT/ $\beta$-catenin signaling plays a critical role in controlling the tumorigenicity and function of transplanted ESC-RPCs. (A) Enriched pathways and gene ontology (GO) of significantly differentially expressed genes between ESC-RPCs and P-RPCs. (B) The fold change in gene expression levels between ESC-RPCs and P-RPCs for components of the WNT signaling pathway. $P<0.05$. (C) The effect of different lengths of time of DKK1 treatment on the outcome of ESC-RPC transplantation. $N$, the number of eyes receiving transplantation in each group. (D) Cryosections of an eyeball containing tumor tissue generated from injected D3EGFP-derived RPCs (green). Scale bar: $100 \mu \mathrm{m}$. GCL, ganglion cell layer; INL, inner nuclear layer; ONL, outer nuclear layer. (E) Immunofluorescence staining for sections of eye tumors produced by D3EGFP-derived RPCs (green) using antibodies against SOX2, N-Cadherin, PAX6, GFAP, TUJ1, Vimentin, NESTIN, fTCF7, and $\beta$-catenin, respectively. The dashed lines indicate the boundaries between recipient tissues and tumors derived from donor cells. Scale bar: $50 \mu \mathrm{m}$.

of 4,488 genes showed differences in expression at the level of 2 -fold and more $(P<0.05)$. These genes primarily encoded molecules associated with neural differentiation, eye development, and WNT signaling (DAVID 6.7; http://david.abcc.ncifcrf.gov) (refs. 25, 26, and Figure 2A). Notably, many genes encoding components of WNT signaling displayed differential expression levels (Figure 2B and Supplemental Table 2). Particularly, high levels of canonical WNT signaling downstream targets (Axin2, Myc, and Ccnd2) and the nuclear distribution of $\beta$-catenin proteins (Supplemental Table 2 and Supplemental Figure 2A) indicated the sustained higher level of WNT signaling in ESC-RPCs compared with that in P-RPCs. To test whether the higher activity of WNT signaling accounted for the higher tumor incidence and poor functional rescue of ESC-RPCs in the SI-induced retina degeneration model, ESC-RPCs were treated with an extracellular inhibitor of WNT signaling (DKK1) (27) for 4 days before transplantation, as 4 days of DKK1 treatment was shown to be the optimal length for achieving the best integration and least tumor formation (Figure 2C). Dramatically, with DKK1 treatment, tumor-free donor cell integration occurred in 58 out of 62 transplanted eyes and tumors were observed in only 2 eyes (Table 1 ). To verify that tumor formation and the function of DKK1 were not specific to 46C ESC-RPCs, we used 3 additional different lines of mouse ESCs, namely a D3 ESC line expressing EGFP constitutively (D3 ${ }^{\text {EGFP }}$ cells), ESCs carrying a Oct4.EGFP transgene (ESC Oct4.EGFP cells) (28), and an ESC line expressing EGFP under the control of the endogenous Sox 1 locus and generated under $2 \mathrm{i}$ condition (2i-ESC ${ }^{\text {SOX1.EGFP }}$ cells) (inhibitors of Fgf/Ras/Mek signaling and GSK3) to generate ESC-RPCs. These ESCs were induced to RPCs according to a recently published protocol for efficient and quick induction of neural retinal cells by floating culture in the presence of Matrigel (ref. 14 and Supplemental Figure 2B). On the seventh day of induction, ESC-derived neural aggregates were digested to single cell suspension. Then, populations of $\mathrm{EGFP}^{+} / \mathrm{NCAM}^{+}, \mathrm{OCT}^{-} / \mathrm{NCAM}^{+}$, and $\mathrm{SOX}^{+} /$ $\mathrm{NCAM}^{+}$neural cells were selected by FACS, respectively (Supplemental Figure 2C). The purpose of this selection was to eliminate pluripotent and nonneural cells. The selected cells were then further differentiated into ESC-RPCs (Supplemental Figure 2D) and tested for their tumorigenicity potential. We found that ESC-RPCs from all 3 lines of ESCs produced tumors at the rate ranging from $56.25 \%$ to $68.75 \%$, with the integration rate from $31.25 \%$ to $37.50 \%$ (Supplemental Table 3). The tumors were developed from injected
ESC-RPCs, as tumor cells expressed EGFP (Figure 2D). They also expressed SOX2, N-Cadherin, PAX6, fTCF1, and NESTIN, revealing the neural nature of tumors (Figure 2E). Remarkably, DKK1 treatment reduced the tumor formation and enhanced integration drastically (Supplemental Table 3). These results are consistent with the transplantation result obtained with 46C ESC-RPCs. Therefore, our data indicate that ESC-RPCs, regardless of the ESC source, induction protocol used, and lentiviral infection, possess the potential to generate tumors following ocular transplantation. Inhibition of canonical WNT signal before cell transplantation is an effective strategy to prevent tumor formation.

Moreover, successful integration and functional rescue of degenerative retina were achieved in DKK1-treated ESC-RPC-transplanted (DKK1-ESC-RPC-transplanted) eyes, as indicated by the following lines of experimental evidence. First, transplantation of DKK1-ESC-RPCs markedly rescued SI-induced reduction in the thickness of the whole retina and loss of the structure of the photoreceptor outer segment (OS) (Figure 3A), which are typical features of retinal degeneration. Second, injected DKK1-ESC$\mathrm{RPCs}^{\mathrm{EGFP}}$ primarily migrated into the outer nuclear layer of the retina and displayed typical morphological features of mature photoreceptors with an outer process, inner segment (IS), and OS (Figure 3, B and C). Third, many integrated $\mathrm{EGFP}^{+}$cells expressed OPSIN, a key molecule for the light transduction cascade (Figure 3C), suggesting their differentiation into rod cells. Last, DKK1ESC-RPCs substantially preserved the scotopic ERG responses $(n=10)$. In comparison, untreated ESC-RPCs only partially preserved the ERG response in the tumor-free eyes $(n=10)$ (Figure 3D). Collectively, these data indicate that inhibition of WNT signaling enabled ESC-RPCs to have the therapeutic capacity closer to that of P-RPCs and that there was a correlation between higher activities of WNT signaling and tumorigenicity of ESC-RPCs.

We next attempted to find what molecular processes took place within DKK1-ESC-RPCs. QRT-PCR data showed that DKK1 repressed the expression of neural progenitor markers but upregulated committed retinal lineage markers, apart from downregulation of many key components of WNT signaling (Supplemental Figure 3A). Immunostaining also showed that percentages of cells positive to BrdU, PCNA, and SOX2 staining (Figure $3 \mathrm{E}$ ) were reduced significantly by DKK1 treatment, whereas fractions of cells expressing MAP2 or OPSIN increased significantly (Supplemental Figure 3B). Clearly, OTX2 ${ }^{+}$or $\mathrm{NRL}^{+}$cells and $\mathrm{NESTIN}^{+}$or BrdU ${ }^{+}$cells were mutually exclusive, although expression of BrdU and NESTIN was found in the same cells (Supplemental Figure 3C), indicating that these $\mathrm{NRL}^{+} / \mathrm{BrdU}^{-} /$ NESTIN $^{-}$cells were fully differentiated postmitotic photoreceptor precursors. The specificity of the antibodies used here was further verified by retinal cross-section immunofluorescence staining of neonatal C57 mice (Supplemental Figure 3D). In addition, FCM analysis revealed reduced percentages of SOX2 ${ }^{+}$ and $\mathrm{PAX}^{+}$cells but increased fractions of $\mathrm{RAX}^{+}$and $\mathrm{CRX}^{+}$cells (Figure 3F). Therefore, DKK1 treatment induced ESC-RPCs into a more P-RPC-like state, which should underline its effect on the functional outcomes of ocular transplantation.

TCF7 mediates the function of canonical WNT signaling in ESC-RPCs. We then asked which factor(s) mediated the functions of canonical WNT signaling in ESC-RPCs. A high level of full-length Tcf7 (fTcf7) was noticed in ESC-RPCs (Figure 4A) and in eye tumors from ESCRPC injection (Figure 4B and Supplemental Figure 4A). Moreover, DKK1 treatment reduced the fractions of fTCF7 ${ }^{+}$and NESTIN $^{+}$ 
A

Normal

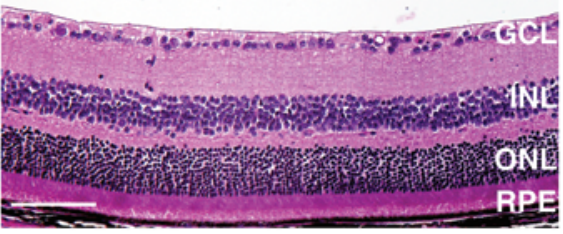

Sham injection

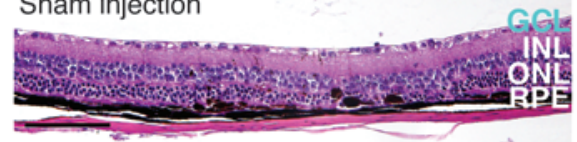

DKK1-ESC-RPC
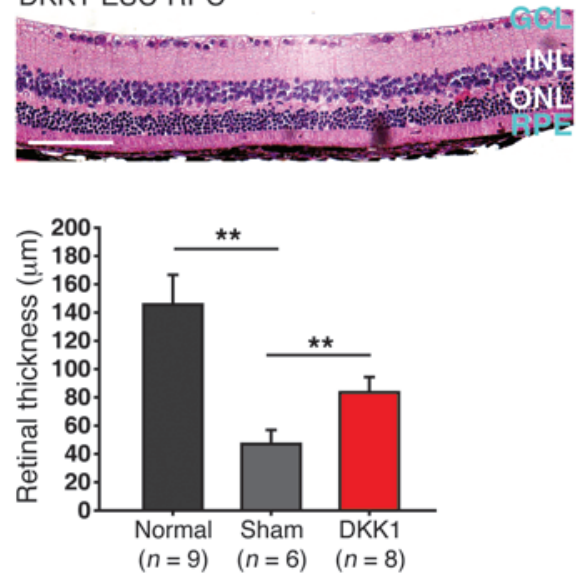

E
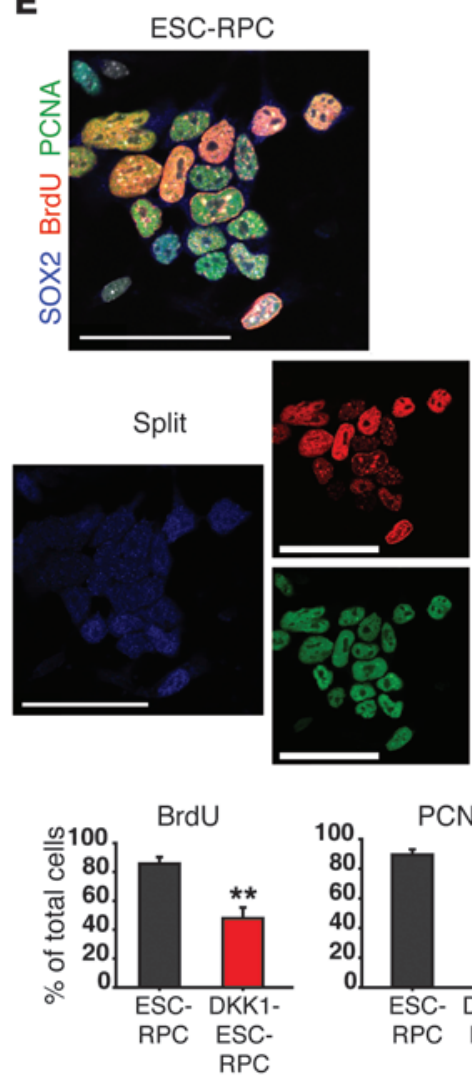

B




F
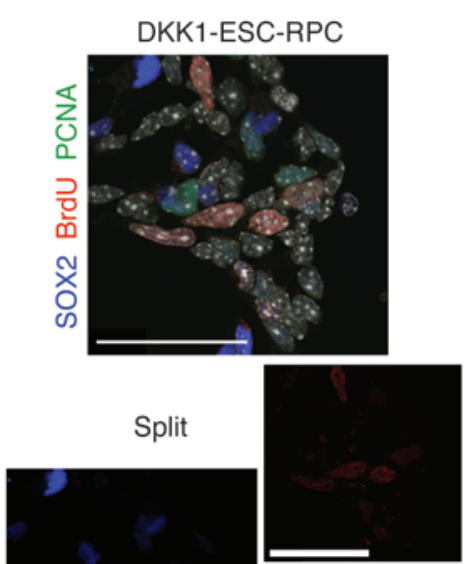

Split
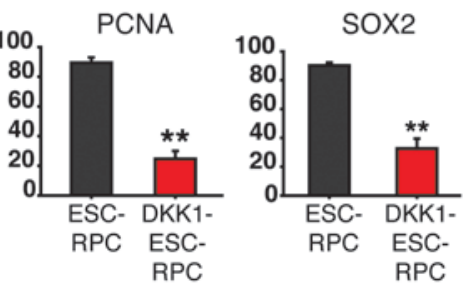

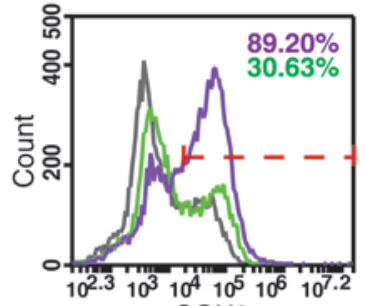

SOX2

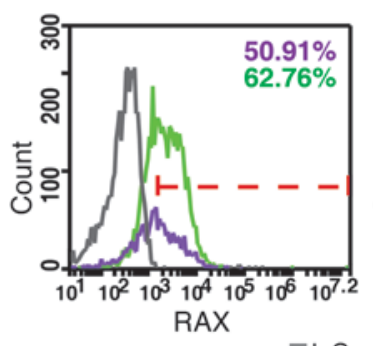

- IgG

-ESC-RPC

DKK1-ESC-RPC
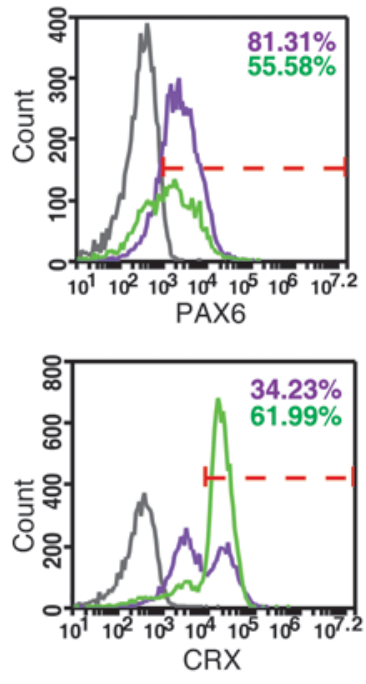

CRX 


\section{Figure 3}

DKK1 treatment drives ESC-RPCs into a more P-RPC-like state. (A) The neural retinal thickness on H\&E-stained sections is shown. The bar chart shows the quantitative analysis of the corresponding staining. $n$, the number of animals; RPE, retinal pigment epithelium. (B) A typical image of integrated DKK1-ESC-RPCsEGFP (green) in the SI-damaged retina. (C) The magnified image of the rectangle shown in $\mathbf{B}$, with OPSIN staining (red). The OPSIN staining is not shown in B for the clarity of donor cell integration. The typical morphology of integrated donor cells: the nucleus (black arrow), outer process (white arrow), IS (white arrowhead), and OS (black arrowhead). (D) Therapeutic effects were monitored by ERG examination. $n=10$ for each group. (E) Immunostaining for proliferation markers and SOX2 in ESC-RPCs with or without DKK1 treatment. Bar charts show the quantitative analysis of the corresponding staining. (F) FCM analysis shows the changes in the percentage of ESC-RPCs expressing SOX2, PAX6, RAX, and $\mathrm{CRX}$, respectively, after DKK1 treatment. IgG was applied as a negative control. DAPI is shown in gray. Data are shown as mean \pm SD; ANOVA (A and D), $t$ test $(\mathbf{E}) ;{ }^{* *} P<0.01$. Scale bar: $50 \mu \mathrm{m}(\mathbf{A}$ and $\mathbf{E})$; $25 \mu \mathrm{m}$ (B and $\mathbf{C})$.

cells in ESC-RPCs significantly (Supplemental Figure 4B). Furthermore, we found that, of TCF/LEF factors, only TCF7 was highly expressed in the developing mouse retina (Supplemental Figure 4, C and D). Based on these observations and considering that $\beta$-catenin usually binds TCF/LEF factors to execute functions of canonical WNT signaling, we anticipated that TCF7 might play a critical role in the control of ESC-RPC differentiation and proliferation, although the implication of TCF7 in neural development and retinal lineage commitment was previously not reported. To test this, a naturally truncated form of TCF7 ( $\triangle \mathrm{NTCF} 7)$, lacking the $\mathrm{N}$ terminus, was used. It produces an inhibitory effect on WNT signaling, as the transcriptional activation activity of TCF/LEF factors is dependent on their association with $\beta$-catenin through the $\mathrm{N}$ terminus (29). The dominant-negative effect of $\triangle$ NTCF7 on canonical WNT signaling was validated by a TOPFlash luciferase assay (Supplemental Figure 4E). At molecular levels, overexpressing $\Delta N T c f 7$ in ESC-RPCs ( $\Delta N T c f 7-E S C$-RPCs) brought about changes resembling those following DKK1 treatment (Figure 4, C and D). At functional levels, engraftment of $\triangle N T c f 7$-ESC-RPCs not only reduced the incidence of tumors to 6 out of 132 eyes but also increased the donor cell integration to 102 out of 132 eyes (Table 1). Transplanted $\Delta N T c f 7$-ESC-RPCs survived and migrated to multiple layers in the host retina. Typical integration and differentiation into rod photoreceptors were indicated by the observation of OS and IS of rod photoreceptors with EGFP expression inside the host retina (Supplemental Figure 4F). In addition, transplantation also preserved ERG in a manner similar to that of DKK1-ESC-RPCs (Figure 4E). Altogether, antagonizing TCF7's activity almost phenocopied the effect of DKK1 treatment both at molecular and visual functional levels.

To further define the role of TCF7 in WNT signaling-mediated tumor formation following ESC-RPC transplantation, a specific Tcf7 RNA interference vector (shTcf7) was introduced into ESCRPCs (Supplemental Figure 4G). Results from FCM analysis indicated that silencing Tcf7 evidently decreased the fraction of SOX2 ${ }^{+}$ and $\mathrm{PAX}^{+}$cells but increased the fraction of $\mathrm{RAX}^{+}, \mathrm{OTX}^{+}$, and $\mathrm{CRX}^{+}$cells, respectively (Figure 5A). Hence, silencing Tcf7 rendered ESC-RPCs more retinal lineage-committed and matured. This conclusion was further supported by transplantation results. Similar to $\triangle N T c f 7$-ESC-RPCs, transplantation of shTcf7-treated ESC-
RPCs gave rise to $69.44 \%$ of successful integration, with tumor formation in $5.56 \%$ of injected eyes (Table 1). This result further strengthened the notion that TCF7 could indeed be the primary mediator of WNT-controlled tumorigenicity of ESC-RPCs.

Last, we tested whether overexpression of fTcf7 could alter the differentiation state of P-RPCs and induce tumor formation in the mouse retina. Exogenous expression of fTcf7 in P-RPCs together with WNT3A treatment enhanced percentages of cells expressing neural progenitor cell (NPC) markers (PCNA, SOX2, and PAX6) substantially, whereas percentages of cells expressing RPC markers (RAX, OTX2, and CRX) decreased markedly (Figure 5B), suggesting that overexpression of fTcf7 switched P-PRCs to a state more like that of NPCs. We subsequently injected lentiviral vectors containing either fTcf7 or EGFP coding sequence together with WNT3A proteins into the retina of P1 mice. Three weeks after the injection, antibodies against glutamine synthetase (a Müller cell marker) and BrdU were used to carry out immunofluorescence staining of retina sections. Disorganized neural tumors were observed in $90 \%$ of eyes injected with fTcf7 lentiviruses and WNT3A, whereas there were no neural tumors detected in the eyes injected with EGFP lentiviruses and WNT3A proteins (Figure 5, C and D, and Supplemental Table 4). Therefore, WNT/TCF7 signaling could induce tumor formation in vivo.

Sox2 and Nestin, direct transcriptional targets of TCF7, control the tumorigenicity and visual repair capability of transplanted ESC-RPCs. In an effort to answer the question of how TCF7 modulated neural differentiation and tumorigenesis, we found that, like TCF7, SOX2 had a high expression level in ESC-RPCs (Figure 1G) and was drastically downregulated by DKK1 treatment (Figure 3, E and F, and Supplemental Figure 3A), $\Delta N T c f 7$ overexpression (Figure 4, C and D), or shTcf7 treatment (Figure 5A), implying a link between TCF7 and SOX2. As SOX2 is known to associate with a large number of tumor types and neural development in vivo (30), we carried out several experiments to test whether TCF7 regulated Sox2 expression directly in ESC-RPCs. Multiple conserved TCF/LEF binding sites were found in the $5 \mathrm{~Kb}$ upstream region of the Sox2 transcription start site (Sox2-5 Kb) (Supplemental Figure 5A). ChIP assays showed the recruitment of TCF7 to the Sox2 promoter under physiological conditions (Figure 6A). EMSAs indicated the direct binding of TCF7 to the Sox2 promoter (Supplemental Figure $5 \mathrm{~B}$ ). Reporter (containing the Sox $2-5 \mathrm{~Kb}$ region) assays revealed a dose-dependent activation of the Sox 2 reporter by fTCF7 in the presence of WNT3A, whereas $\triangle$ NTCF7 abrogated the active effect of fTCF7 (Supplemental Figure 5C). These results clearly demonstrated that Sox2 is a direct downstream target of TCF7. In addition to TCF7, known WNT signaling agonists (WNT3A and $\mathrm{LiCl}$ ) and $\beta$-catenin (Supplemental Figure 5D and Figure 6B) significantly activated the Sox2 reporter activity, indicating a regulatory role of canonical WNT signaling over Sox 2 .

To investigate the role of SOX2 in controlling the property of ESC-RPCs, its expression was stably silenced by a Sox2-specific RNA interference construct (shSox2) (Supplemental Figure 5E). Immunofluorescence staining and qRT-PCR results showed that silencing of Sox2 significantly reduced the expression of neural progenitor-associated genes but increased the transcription of genes associated with neural retinal lineages (Figure 6C and Supplemental Figure 5, F and G). Notably, the expression of WNT signaling components, such as Ctnnb1, fTcf7, and Axin2, was also repressed to various degrees, implying a possible feedback loop between SOX2 and WNT signaling. 

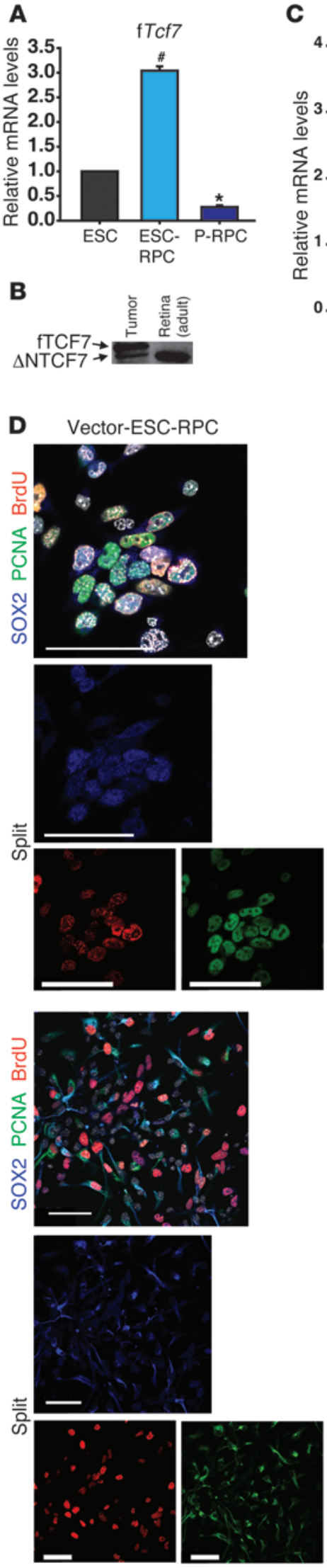

C



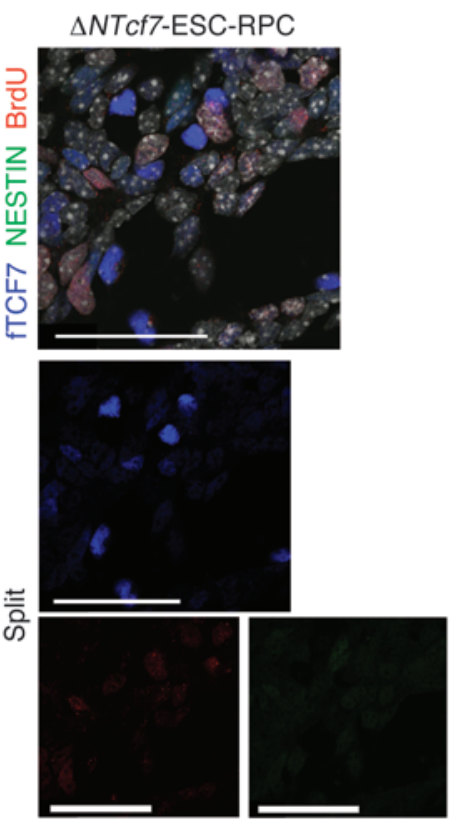
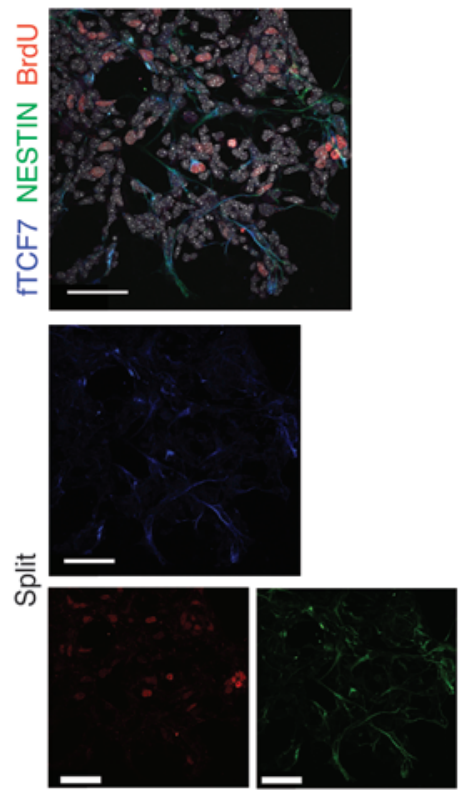
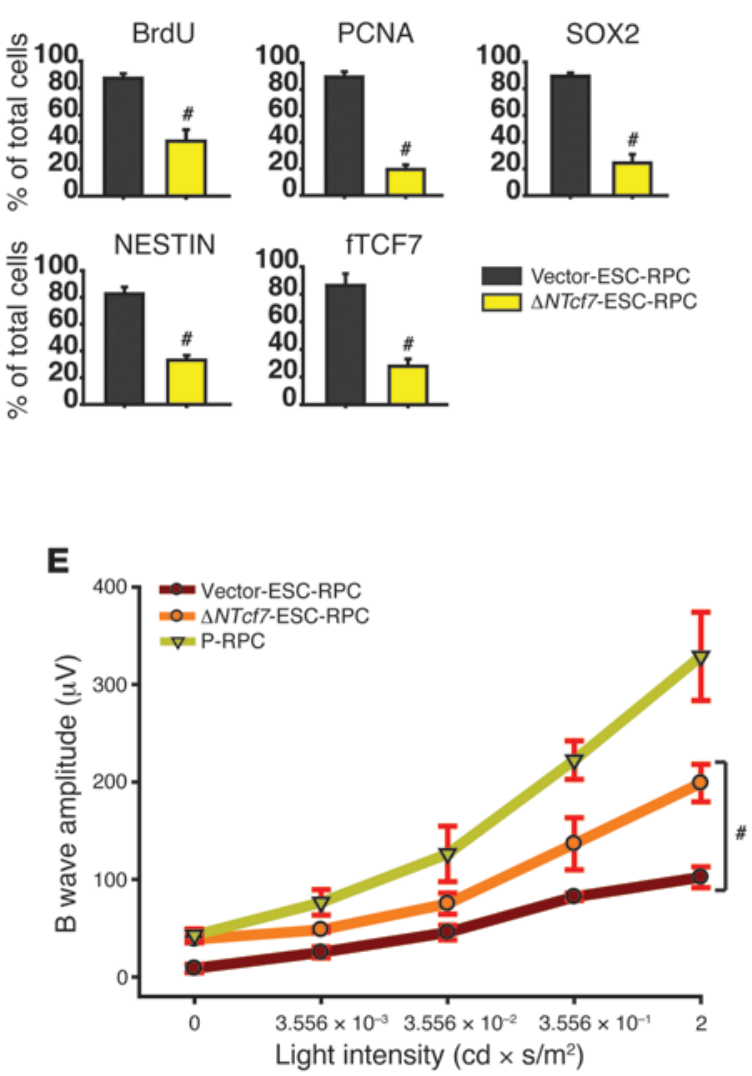

Figure 4

TCF7 mediates functions of WNT signaling in transplanted ESC-RPCs. (A) The comparison of $f$ Tcf7 mRNA levels among ESCs, ESC-RPCs, and P-RPCs by qRT-PCR analysis. Values in ESCs were set at 1.0. (B) Protein levels of TCF7 in ESC-RPCEGFP-derived tumors and normal adult retinae were determined by Western blot analysis. (C) The relative expression levels of neural progenitor markers and WNT signaling in ESC-RPCs, $\triangle N T c f 7-E S C-R P C s$, and P-RPCs were determined by qRT-PCR. Values in empty vector-infected ESCRPCs were set at 1.0. (D) Effects of $\triangle N T c f 7$ on the expression of neural progenitor and proliferation markers in ESC-RPCs were analyzed. Bar charts show the quantitative analysis. Scale bar: $50 \mu \mathrm{m}$. (E) ERG results for eyes grafted with empty vector-infected ESC-RPCs, $\triangle N T c f 7-E S C-R P C s$, and P-RPCs. $n=10$ for each group. Data are shown as mean \pm SD; ANOVA (A, C, and E), $t$ test (D); ${ }^{*} P<0.05,{ }^{\#} P<0.01$. 

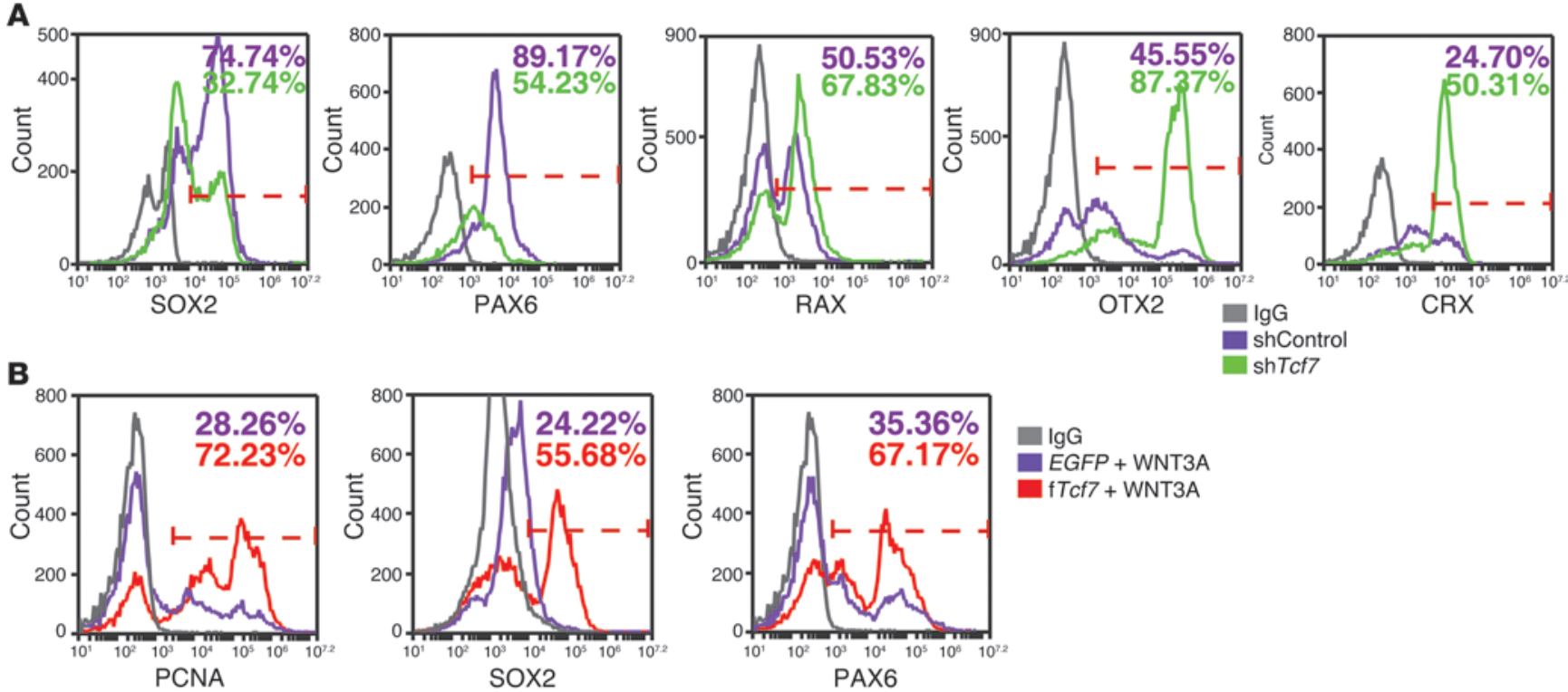

shContro
shTcf7


C

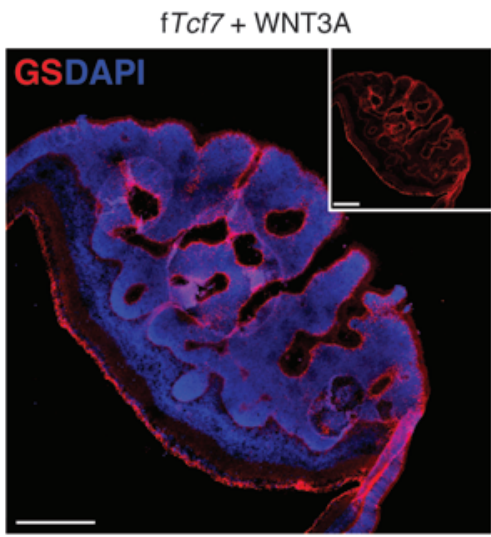

D $E G F P+$ WNT3A
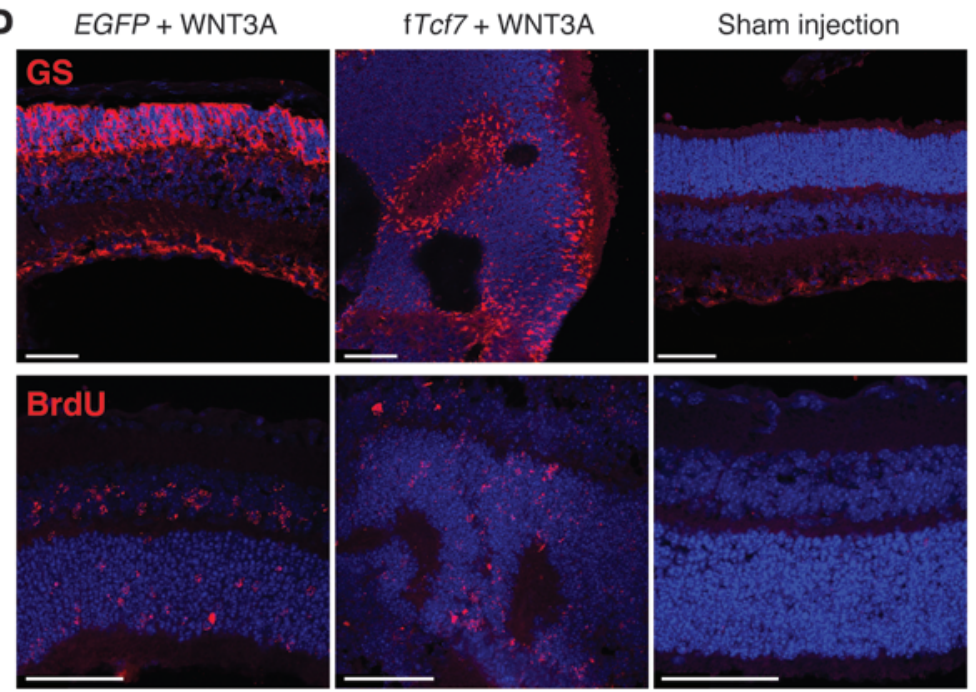

Figure 5

The expression level of TCF7 is directly linked to the state of ESC-RPCs and eye tumor formation in vivo. (A) FCM analysis to compare the percentage of ESC-RPCs expressing SOX2, PAX6, RAX, OTX2, and CRX, respectively, between shControl and shTcf7-ESC-RPCs. (B) FCM analysis to compare the percentage of P-RPCs expressing PCNA, SOX2, PAX6, RAX, OTX2, and CRX, respectively, between vector-infected and $\mathrm{fT}$ cf7-infected P-RPCs in the presence of WNT3A protein. (C and D) Immunofluorescence staining with antibodies against glutamine synthetase (GS) and BrdU in retinae injected with lentiviral fTCF7 and WNT3A protein. Retinae injected with lentiviral EGFP plus recombination WNT3A protein and the medium were used as controls. Scale bar: $50 \mu \mathrm{m}$ (C); $250 \mu \mathrm{m}$ (D). 
A
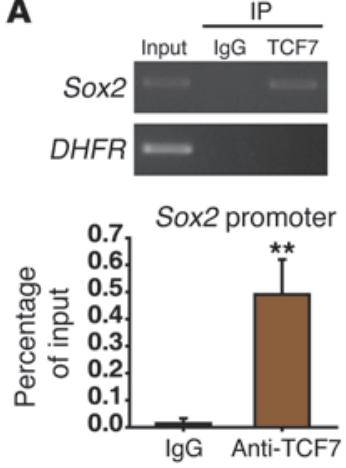

B

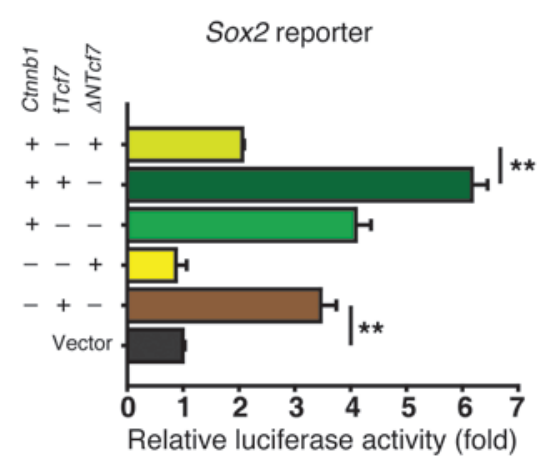

C
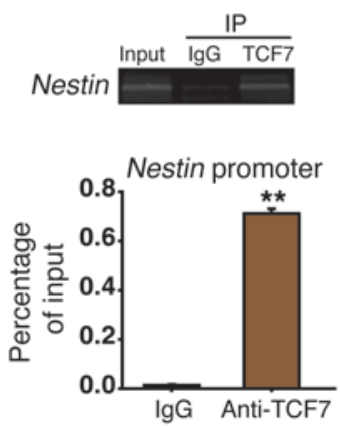

D



shSox2

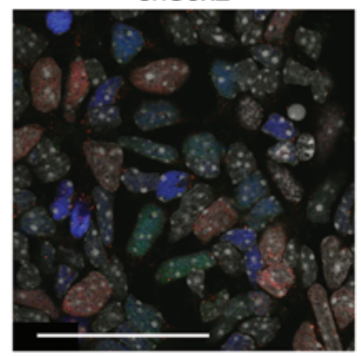

shControl


E

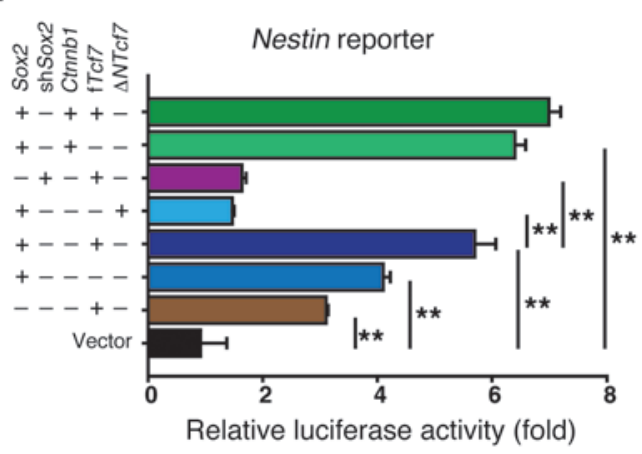

$\mathbf{F}$

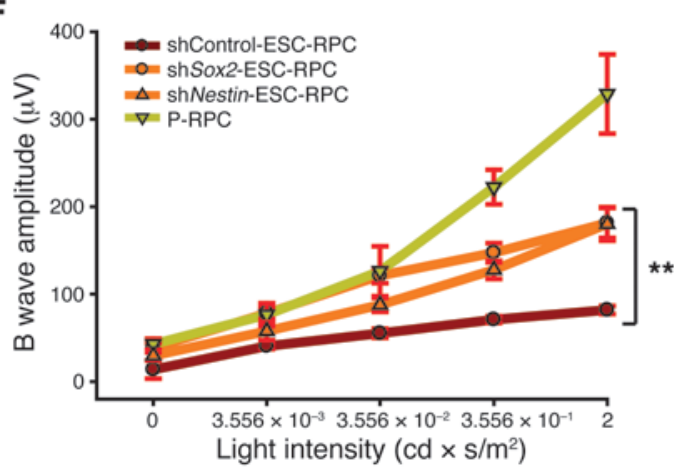




\section{Figure 6}

SOX2 and NESTIN act as direct targets of TCF7, determining functional consequences of ESC-RPC transplantation. (A) ChIP assays were performed using rabbit IgG or the specific antibody against TCF7 in ESC-RPCs. The locus of DHFR was used as a negative control. (B) TCF7 and $\beta$-catenin cooperatively transactivated the Sox 2 promoter. (C) Effects of shSox2 on immunostaining for proliferation and neural progenitor markers in ESC-RPCs. DAPI is shown in gray. Bar charts show the quantitative analysis of the corresponding staining. (D) ChIP assay results are shown. (E) The effect and interaction of TCF7, SOX2, and $\beta$-catenin on the activation of the Nestin promoter was examined using luciferase reporter assays. (F) ERG analysis of SI-treated mice after transplantation with shSox2- and shNestin-ESC-RPCs EGFP. $n=10$ for each group. Data are shown as mean \pm SD; $t$ test $(\mathbf{A}, \mathbf{C}$, and D), ANOVA (B, E, and F); ${ }^{* \star} P<0.01$. Scale bar: $50 \mu \mathrm{m}$ (C).

In addition, we found 2 evolutionarily conserved TCF/LEF binding sites in the second intron of the Nestin gene (Supplemental Figure 6A). NESTIN was previously reported as a direct target of SOX2 $(31,32)$ and has been recently suggested as a potential marker for cancer stem cells, particularly for cancers of neuroectodermal origin (33). Data from ChIP, EMSA, and reporter assays all demonstrated that TCF7 positively regulated Nestin expression through direct association with Nestin's second intron (Figure 6D and Supplemental Figure 6, B and C). Further reporter assays showed that WNT3A and $\mathrm{LiCl}$ also activated Nestin expression (Supplemental Figure 6D). A stronger activation of the Nestin reporter was detected when SOX2 was coexpressed with $\beta$-catenin or fTCF7. In contrast, the effect of SOX2 on activation of the Nestin reporter was blocked by $\triangle$ NTCF7, and the activation effect of TCF7 was abolished by shSox2 expression (Figure 6E), revealing a functional link between SOX2 and TCF7/ $\beta$-catenin complexes. To verify the interaction among TCF7, $\beta$-catenin, and SOX2 proteins, coimmunoprecipitation and coimmunofluorescence staining assays were carried out. Results from both assays supported the notion that TCF7, $\beta$-catenin, and SOX2 form protein complexes to collaboratively regulate Nestin expression (Supplemental Figure 6, E and F).

The above results established the transcriptional regulation links among TCF7/ $\beta$-catenin, SOX2, and NESTIN. We next determined whether SOX2 and NESTIN play a role in the control of tumor formation and retina repair. To this end, ESC-RPCs transfected with a Sox or Nestin-specific RNA interference construct (shNestin) were transplanted into the same mouse model, respectively, as described above. Like in the case of $\Delta N T c f 7$-ESC-RPCs, markedly higher rates of donor-cell integration $(65 \%$ and $70 \%$ for shSox2 and shNestin, respectively) and lower incidence of tumor formation (5\% and 13\% for shSox 2 and shNestin, respectively) were obtained as compared with control ESC-RPCs transfected with a control RNA interference construct (shControl) (Table 1). Functionally, ERG analysis revealed improved $b$ wave amplitudes in the eyes in which shSox2 or shNestin-ESC-RPCs integrated successfully, while no measurable response was detected in mice of the sham group (Figure 6F). Therefore, similar to DKK1 treatment and $\Delta N T c f 7$ overexpression, the silencing of Sox 2 or Nestin markedly reduced tumorigenesis and improved visual function, although their effects were relatively less dramatic than DKK1 and $\triangle$ NTCF7. Thus, as direct transcription targets of TCF7, SOX2 and NESTIN play crucial roles in determining the properties of ESCRPCs and functional outcomes following transplantation.
TCF7, SOX2, and NESTIN are closely associated with the mouse retina early development. Having shown above that TCF7, SOX2, and NESTIN all play important roles in determining the effects of transplanted ESC-RPCs, we were interested in knowing whether their expression pattern was associated during early retinal development. To address this issue, we examined their temporal and spatial expression patterns using a Nestin enhancer-driven (second intron) EGFP expression mouse model (32). As illustrated by fluorescence staining in the whole retina on $\mathrm{P} 1$ and $\mathrm{P} 3$ (the peak of rod photoreceptor production) (34), fTCF7-expressing cells and Nestin-driven $\mathrm{EGFP}^{+}$cells were seen in the whole retina, whereas SOX2-expressing cells were detected in the inner layer of the retina. At P7, signals for all 3 factors decreased markedly and fTCF7 expression was restricted to the cells in the inner layer. At P14 and P30, only weak signals could be detected for these 3 proteins (Figure 7A). Concordantly, Western blot analysis indicated that TCF7, SOX2, NESTIN, and PCNA were all highly expressed in the retina at embryonic week 2, P1, and P3 but undetectable at later developmental stages and in adults. Intriguingly, $\triangle$ NTCF7 displayed a reciprocal pattern to that of fTCF7, suggesting the importance of balance between fTCF7 and $\triangle$ NTCF7 for retinal development. The decrease of fTCF7 and increase of $\triangle$ NTCF7 expression were concomitant with the reduction in SOX2 and NESTIN expression as well as retinal cell maturation. The protein level of $\beta$-catenin, however, remained unchanged during all stages tested (Figure 7B). Parallel expression patterns of TCF7, SOX2, and NESTIN suggest that they might share expression regulation and have related functions during retinal development.

Therefore, the WNT-linked TCF7-SOX2-NESTIN cascade might be closely associated with biological properties of both normal neural progenitors and neural tumor cells.

\section{Discussion}

In this study, through functional evaluation of multiple kinds of ESC-RPCs in comparison with P-RPCs in a retinal degeneration model (Table 1) and genome-wide transcriptome analyses, we report that the sustained activation of the canonical WNT signaling-coupled TCF7 $/ \beta$-catenin-SOX2-NESTIN cascade contributed to tumorigenesis in the ocular transplantation of ESC-RPCs. Inhibition of the cascade in ESC-RPCs prior to transplantation gave rise to a drastic reduction in tumor formation and substantial improvement in visual preservation after transplantation in the mouse retinal degeneration model (Figure 7C). This finding is of particular significance, given that tumor formation is one of the major obstacles for clinical application of ESC-derived cells, and emphasizes the importance of controlling and monitoring donor cell status for successful transplantation.

Another impact of this study is the uncovering of a crucial role for TCF7 in neural and eye development. TCF7 is best known for its function in $\mathrm{T}$ cell development (35). Our data discovered its previously unrecognized expression pattern and critical roles in early eye development as well as RPC differentiation/proliferation regulation, particularly for safe and effective transplantation of ESC derivatives. So far, there is no clear answer yet as to whether TCF7 acts as a WNT-linked tumor suppressor or a tumor promoter. Mice deficient in the Tcf7 gene developed intestinal and mammary adenomas, suggesting a role of tumor suppressor (17), while our results argue that fTCF7 acts as a tumor promoter in the context of ESC-RPC transplantation. Overall, the outcome of $T c f 7$ deletion should depend on the cell type and the developmen- 

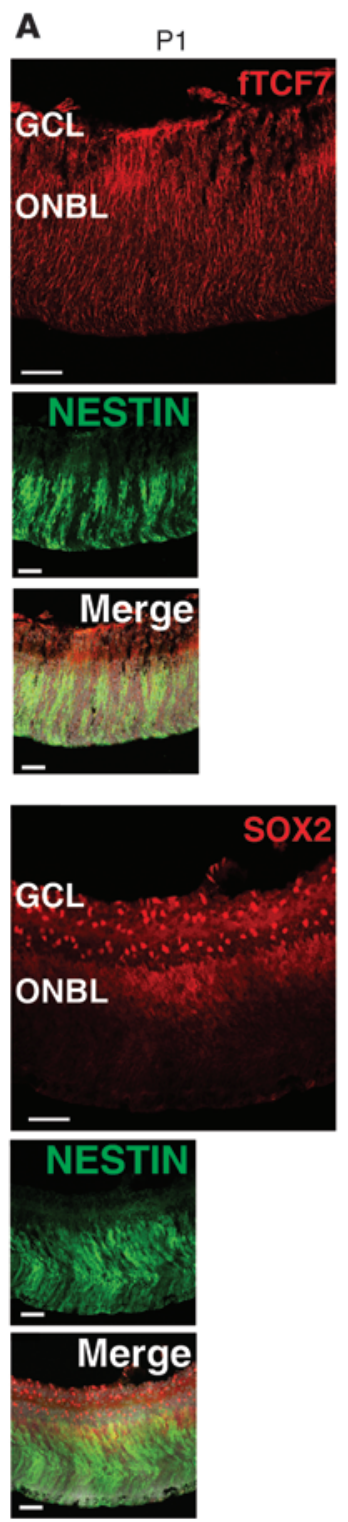

B ק-Catenin

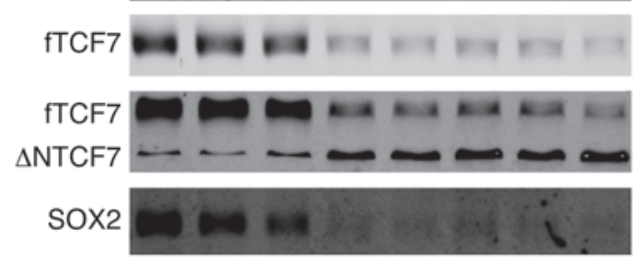

NESTIN

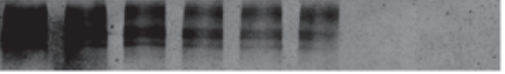

PCNA

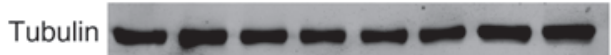

P7
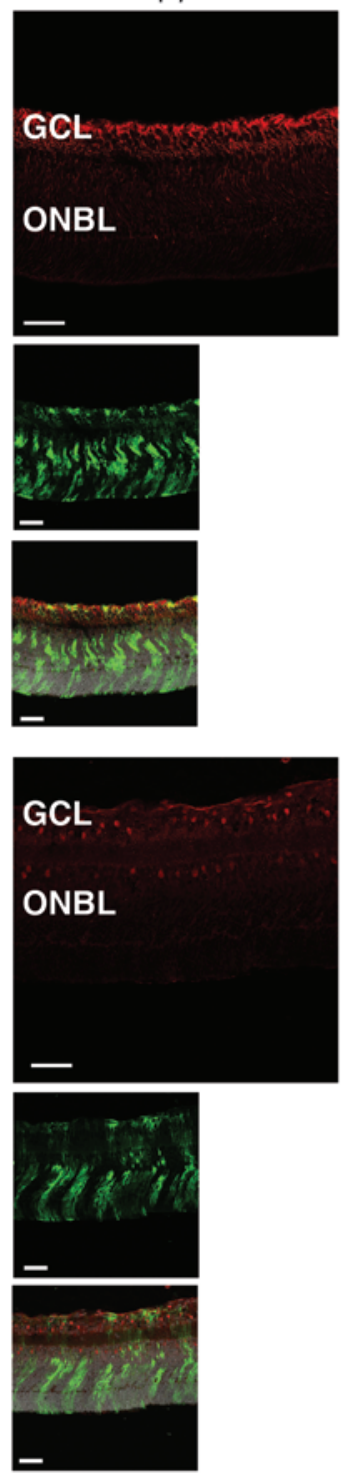

C
P14
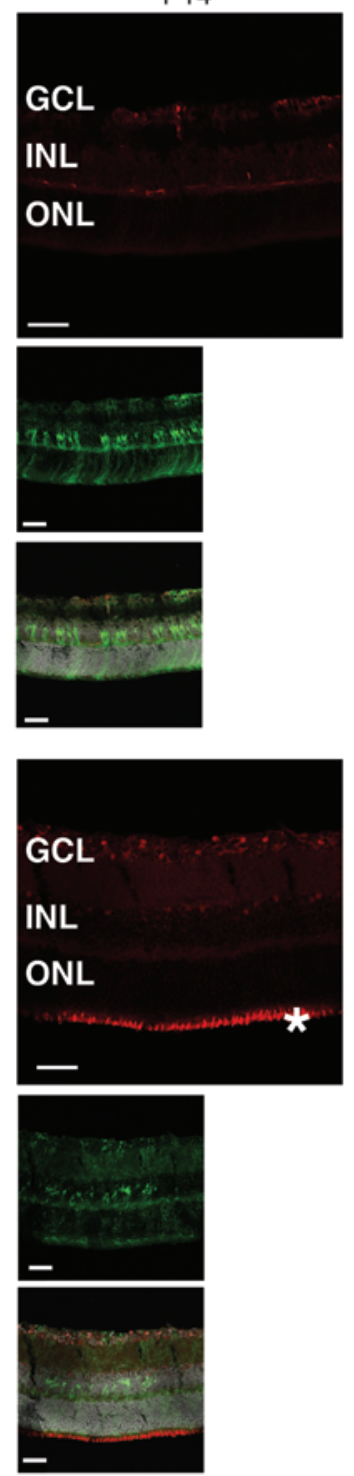

P30


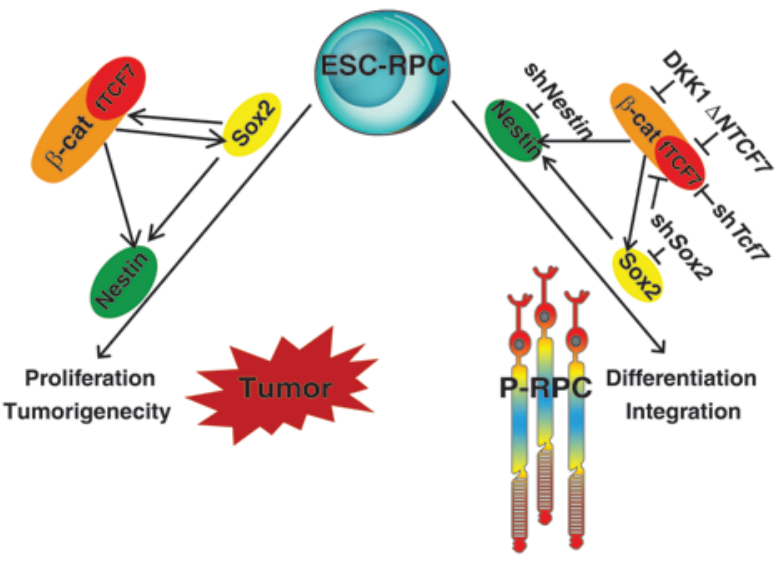




\section{Figure 7}

Canonical WNT signaling does not only define the cell fate of transplanted ESC-derived retinal progenitors but is also closely associated with early development of the mouse retina. (A) Immunofluorescence staining for the expression of fTCF7 and SOX2 in the retina of a Nestin. EGFP transgenic mouse from $\mathrm{P} 1$ to $\mathrm{P} 30$. DAPI is shown in gray. The asterisks indicate the nonspecific staining. ONBL, outer neuroblastic layer. Scale bar: $50 \mu \mathrm{m}$. (B) Representative Western blots of $\beta$-catenin, fTCF7, TCF7, SOX2, NESTIN, and PCNA proteins in mouse retina from E14 to adult. (C) The proposed model explains how canonical WNT signaling determines the proliferation and differentiation state of ESC-derived progenitors via TCF7 to regulate the expression of Sox2 and Nestin in vitro, controlling tumorigenesis and functional visual preservation in a disease model. The potential targets to block the pathway are indicated. $\beta$-cat, $\beta$-catenin.

tal stage studied, i.e., whether the full-length or truncated form of TCF7 is dominantly expressed as well as whether there are other members of the TCF/LEF family coexpressed. For instance, in the intestine, the most abundant TCF7 isoform is $\triangle \mathrm{NTCF} 7$, a repressor of WNT signaling (17), and TCF7L2 is coexpressed. Deletion of Tcf7 would lead to increased expression of $\beta$-catenin/TCF7L2 target genes and the development of adenomas. Differently, fTCF7 is the dominant isoform expressed in embryonic retinae and ESCRPCs. Silencing of Tcf7 would reduce the activity of WNT signaling and tumor formation. Therefore, we propose that the balance between expression levels of full-length and truncated forms of TCF7 is a key to determine the state of RPCs. In addition, we propose that WNT-activated TCF7 controls the state of ESC-RPCs by directly regulating Sox2 and Nestin expression. Although the regulation of SOX 2 expression by WNT/ $\beta$-catenin/TCF signaling in the developing Xenopus retina was previously reported (36), our identification of the direct link between TCF7 and its downstream targets (Sox2 and Nestin) as well as protein complexes consisting of TCF7, $\beta$-catenin, and SOX2 provides molecular insights into how WNT signaling modulates the cell fate in neural stem cells/NPCs.

Nevertheless, it is a possibility that other signaling pathways and intrinsic factors are also involved in the regulation of the ESC-RPC state. It is noteworthy that inhibition of the TCF7/ $\beta$-catenin-SOX2NESTIN cascade drove ESC-RPCs into a developmental stage and cell type components similar to those of P-RPCs. However, they were not the same. For example, the percentages of cells expressing PCNA and PAX6 in DKK1- or shTcf7-ESC-RPCs were moderately higher than in P-RPCs, although the fractions of cells expressing SOX2, OTX2 and CRX were similar. The difference also manifested as a few incidences of tumor formation as well as the lower efficiency of visual preservation in DKK1-ESC-RPC transplantations compared with that in P-RPCs. To identify pathways that might underlie the phenotypic differences between P-RPCs and DKK1-ESC-RPCs, we compared the transcriptomes between P-RPCs and DKK1-ESCRPCs. Intriguingly, many components of the ERK/MAPK pathway were differentially expressed between the 2 types of cells (Supplemental Figure 7 and Supplemental Table 5). The transcript levels for certain activators of ERK/MAPK signaling, such as Fgf1, Fgf4, Fgfr1, Fgfr2, Fgfr3, and Kras, were markedly higher in DKK1-ESC-RPCs than in P-RPCs, suggesting possible association of the ERK/MAPK pathway with the residual problem of eye tumor development. Further investigations are warranted to eliminate tumor formation entirely.

Recently, West et al. demonstrated the optimized generation of retinal photoreceptor precursors from mouse ESCs and evaluated the transplantation competence of retinal populations at different stages of development in healthy and degenerating mouse retinae. They observed tumors containing neural morphology and rosettes after transplantation of ESC-derived retinal cells into the subretinal space of mice at day 10 and day 20 of differentiation, even after sorting using a Rax.GFP transgene (6). They also showed that cells from day 28 of the differentiation protocol did not give rise to any tumors. However, these cells did not have the ability to integrate into host retinae. Their finding is consistent with the general opinion that extended differentiation can reduce or abolish tumor formation following transplantation of ESC-derived cells. However, it is also true that prolonged differentiation deprives donor cells of the ability to functionally integrate and rescue the disease phenotype. Therefore, it is critically important to define and monitor the appropriate stage of ESC derivatives at molecular and cellular levels for their safe and effective transplantation, regardless of the differentiation procedures used. In this study, a SFEB-based chemical defined differentiation strategy was used. Unlike previously reported protocols, we selected SOX1.EGFP-positive NPCs first and added small molecule inhibitors afterward to induce efficient photoreceptor precursor production. The ESC-RPCs generated through this protocol resulted in a high rate of neural tumor formation following ocular injection, which could be ascribed to the sustained high level of canonical WNT signaling-activated TCF7/ $\beta$-catenin-SOX2-NESTIN cascade in donor ESC-RPCs. Indeed, we found that expression levels of fTcf7, Sox2, and Nestin were significantly higher in ESC-RPCs than in P-RPCs. The ESCRPCs might be more similar to embryonic RPCs. Strikingly, DKK1 treatment at day 24 of differentiation for 4 days rendered ESCRPCs more like P-RPCs, demonstrating that inhibition of canonical WNT signaling at the late stage of differentiation favors the generation of P-RPC-like cells. Importantly, our results show that the development of neural tumors and their elimination by DKK1 treatment are not specific to the RPCs derived from 46C ESCs but are instead independent of ESC sources. On the other hand, nearly complete elimination of tumor formation by DKK1 treatment rules out the possibility that tumor formation after ESC-RPC transplantation was due to the development of genetic alterations during in vitro culture. Similarly, MacLaren and colleagues found that the embryonic immature progenitors formed rosettes in the subretinal space, whereas mature retinal cells did not survive well upon transplantation (7). Therefore, the developmental stage of donor cells critically determines their fates following transplantation. Here, driving ESC-RPCs into a P-RPC-like state by DKK1 and shTcf7 offers an attractive strategy to obtain cells at the appropriate state from a renewable cell source.

The SI model was chosen in this study because it better mimics human degenerative retina diseases, including age-related macular degeneration, a leading cause of blindness in the world. Similar to pathological changes in age-related macular degeneration, SI selectively damages retinal pigment epithelial cells, leading to the secondary degeneration of photoreceptors. Furthermore, to surmount the problem of the individual variance in response to SI treatment and ESC-RPC transplantation, a large number of mice were used in this study. Successful transplantation of P-RPCs in the SI-induced retinal degeneration model validates the appropriateness of the experimental procedures used here and highlights the promising future of cell-replacement therapy. The obvious visual preservation obtained from DKK1-, $\Delta N T c f 7-$, shSox2-, and shNestin-treated ESC-RPCs could be partially attributed to the 
survival and differentiation into photoreceptors of donor cells, as indicated by the expression of rod photoreceptor markers in $\mathrm{EGFP}^{+}$donor cells, although the integrated cell number was small. Previous studies reported that even a relatively small number of transplanted retinal cells can be sufficient to rescue visual function and that some vision is retained in patients having few photoreceptors (37). On the other hand, the impact of transplanted cells on the remaining host retinal cells should play a role as well, since we observed significant recovery in the thickness of different layers of host retinae, even for the layer in which few donor cells could be detected. Secreted soluble factors, which have previously been implicated in the mechanism for visual rescue by human NPCs (38), could be partially responsible for the improvement of retinal functions. Meanwhile, excessive proliferation or dedifferentiation of immature donor cells due to their response to local growth signals might also contribute to tumor formation after transplantation. The precise mechanisms underlying the morphological and functional recovery as well as the development of neural tumors following ocular transplantation of ESC-RPCs warrant further investigation. Overall, this study provides the proof of principle for optimizing the activity of extracellular signaling and related intrinsic factors prior to donor cell transplantation to prevent tumor formation and improve therapeutic effects.

\section{Methods}

SI-induced retinal degeneration model and subretinal transplant surgery. Experimental C57BL/6 mice at 8 weeks of age were used to induce a retinal degeneration model by a single intravenous injection of $30 \mathrm{mg} / \mathrm{kg} \mathrm{SI}$ (Sigma-Aldrich) (20). Injected cells were suspended in the GMEM at the density of $8 \times 10^{4} / \mu \mathrm{l}$ (Gibco). Then, cell suspensions or enriched lentiviruses $(1 \mu \mathrm{l})$ were transferred into subretinal space in a manner similar to the one previously described (7).

ERG examination. Corneal ERG recordings from both eyes of mice were obtained 3 weeks after cell transplantation. ERG recordings were performed with an AVES system (Kanghua Rui Ming Technology Co. Ltd.) following the procedures described previously (39).

Microarray analyses. RNA samples from 3 independent experiments were hybridized to a whole mouse gene expression microarray (Affymetrix Mouse 430 2.0) following the manufacturer's instruction. For each sample, the background was removed, and data were normalized in accordance with
MAS 5.0. A hierarchical clustering of samples was performed using Cluster 3.0 software (Michael Eisen, Stanford University). Heat maps were generated using Java Treeview software (http://jtreeview.sourceforge.net; under GPL).

Accession number. Microarray data are accessible at the GEO database under accession number GSE34002.

Statistics. Data were pooled from at least 3 independent sets of experiments, unless otherwise indicated, and are presented as the mean \pm SD. All statistical analyses were carried out using SPSS 11 (SPSS). Comparisons of mean values were analyzed by Student's $t$ test ( 2 sided) or 1-way ANOVA followed by the post-hoc Dunnett's test for experiments with more than 2 groups (Levene's tests for equal variance). Dunnett's T3 test was used as post-hoc test comparison for the analysis of unequal variances (Welch's and Brown-Forsythe's test). Differences were considered statistically significant at $P<0.05$. Graphing was performed using SigmaPlot software.

Study approval. All animal procedures and experiments were approved by the Shanghai Jiao Tong University School of Medicine Animal Care Committees. Animals were cared for in accordance with the Association of Research for Vision and Ophthalmology statement for the use of Animals in Ophthalmic and Vision Research.

\section{Acknowledgments}

We thank Juan Carlos Izpisua Belmonte and Lin Li for valuable suggestions and Erbo $\mathrm{Xu}$ for critical reading of the manuscript. This work was supported by grants from the National High Technology Research and Development Program of China (2010CB945200, 2011DFB30010, 2011CB965101, 2013CB966801, and 2009CB941103), National Natural Science Foundation (91019023, 31000639, and 31171419), and Chinese Academy of Science (XDA01010102). This study was also supported by the Shanghai Leading Academic Discipline Project (S30201).

Received for publication May 29, 2012, and accepted in revised form January 17, 2013.

Address correspondence to: Guo-Tong Xu, Medical Building, Room 521, 1239 Siping Road, Shanghai, 200092, China. Phone: 0086.21.6598.6358; Fax: 0086.21.6598.6358; E-mail: gtxu@tongji. edu.cn. Or to: Ying Jin, Room 607, Building 1, 225 South Chongqing Road, Shanghai 200025, China. Phone: 0086.21.63852591; Fax: 0086.21.63852591; E-mail: yjin@sibs.ac.cn.
1. Arnhold S, Klein H, Semkova I, Addicks K, Schraermeyer U. Neurally selected embryonic stem cells induce tumor formation after long-term survival following engraftment into the subretinal space. Invest Ophthalmol Vis Sci. 2004;45(12):4251-4255.

2. Wang NK, et al. Transplantation of reprogrammed embryonic stem cells improves visual function in a mouse model for retinitis pigmentosa. Transplantation. 2010;89(8):911-919.

3. Seminatore $C$, et al. The postischemic environment differentially impacts teratoma or tumor formation after transplantation of human embryonic stem cell-derived neural progenitors. Stroke. 2010; 41(1):153-159.

4. Tucker BA, et al. Transplantation of adult mouse iPS cell-derived photoreceptor precursors restores retinal structure and function in degenerative mice. PLoS One. 2011;6(4):e18992.

5 . Doi D, et al. Prolonged maturation culture favors a reduction in the tumorigenicity and the dopaminergic function of human ESC-derived neural cells in a primate model of Parkinson's disease. Stem Cells. 2012;30(5):935-945.

6. West EL, et al. Defining the integration capacity of embryonic stem cell-derived photoreceptor precur- sors. Stem Cells. 2012;30(7):1424-1435.

7. MacLaren RE, et al. Retinal repair by transplantation of photoreceptor precursors. Nature. 2006; 444(7116):203-207.

8. Pearson RA, et al. Restoration of vision after transplantation of photoreceptors. Nature. 2012; 485(7396):99-103.

9. Lamba DA, Karl MO, Ware CB, Reh TA. Efficient generation of retinal progenitor cells from human embryonic stem cells. Proc Natl Acad Sci U S A. 2006; 103(34):12769-12774.

10. Hirami Y, et al. Generation of retinal cells from mouse and human induced pluripotent stem cells. Neurosci Lett. 2009;458(3):126-131.

11. Meyer JS, et al. Optic vesicle-like structures derived from human pluripotent stem cells facilitate a customized approach to retinal disease treatment. Stem Cells. 2011;29(8):1206-1218.

12. Ikeda $\mathrm{H}$, et al. Generation of $\mathrm{Rx}^{+} / \mathrm{Pax} 6+$ neural retinal precursors from embryonic stem cells. Proc Natl Acad Sci U S A. 2005;102(32):11331-11336.

13. Osakada F, et al. Toward the generation of rod and cone photoreceptors from mouse, monkey and human embryonic stem cells. Nat Biotechnol. 2008; 26(2):215-224.
14. Eiraku M, et al. Self-organizing optic-cup morphogenesis in three-dimensional culture. Nature. 2011;472(7341):51-56.

15. Clevers H. Wnt/beta-catenin signaling in development and disease. Cell. 2006;127(3):469-480.

16. Clevers H, Nusse R. Wnt/beta-catenin signaling and disease. Cell. 2012;149(6):1192-1205.

17. Roose J, et al. Synergy between tumor suppressor APC and the beta-catenin-Tcf4 target Tcf1. Science. 1999;285(5435):1923-1926.

18. Inoue $\mathrm{T}$, et al. Activation of canonical Wnt pathway promotes proliferation of retinal stem cells derived from adult mouse ciliary margin. Stem Cells. 2006;24(1):95-104.

19. Osakada F, Ooto S, Akagi T, Mandai M, Akaike A, Takahashi M. Wnt signaling promotes regeneration in the retina of adult mammals. J Neurosci. 2007;27(15):4210-4219.

20. Kiuchi K, Yoshizawa K, Shikata N, Moriguchi K, Tsubura A. Morphologic characteristics of retinal degeneration induced by sodium iodate in mice. Curr Eye Res. 2002;25(6):373-379.

21. Enzmann V, et al. Behavioral and anatomical abnormalities in a sodium iodate-induced model of retinal pigment epithelium degeneration. Exp 
Eye Res. 2006;82(3):441-448.

22. Aubert J, et al. Screening for mammalian neural genes via fluorescence-activated cell sorter purification of neural precursors from Sox1-gfp knock-in mice. Proc Natl Acad Sci U S A. 2003;100(suppl 1):11836-11841.

23. Ying QL, Stavridis M, Griffiths D, Li M, Smith A. Conversion of embryonic stem cells into neuroectodermal precursors in adherent monoculture. Nat Biotechnol. 2003;21(2):183-186.

24. Okabe M, Ikawa M, Kominami K, Nakanishi T, Nishimune Y. 'Green mice' as a source of ubiquitous green cells. FEBS Lett. 1997;407(3):313-319.

25. Huang da W, Sherman BT, Lempicki RA. Bioinformatics enrichment tools: paths toward the comprehensive functional analysis of large gene lists. Nucleic Acids Res. 2009;37(1):1-13.

26. Huang da W, Sherman BT, Lempicki RA. Systematic and integrative analysis of large gene lists using DAVID bioinformatics resources. Nat Protoc. 2009;4(1):44-57.
27. Nusse R. Developmental biology. Making head or tail of Dickkopf. Nature. 2001;411(6835):255-256.

28. Yang H, et al. Generation of genetically modified mice by oocyte injection of androgenetic haploid embryonic stem cells. Cell. 2012;149(3):605-617.

29. Waterman ML. Lymphoid enhancer factor/T cell factor expression in colorectal cancer. Cancer Metastasis Rev. 2004;23(1-2):41-52.

30. Gangemi RM, et al. SOX2 silencing in glioblastoma tumor-initiating cells causes stop of proliferation and loss of tumorigenicity. Stem Cells. 2009;27(1):40-48.

31. Jin $Z$, et al. Different transcription factors regulate nestin gene expression during P19 cell neural differentiation and central nervous system development. J Biol Chem. 2009;284(12):8160-8173.

32. Tanaka S, Kamachi Y, Tanouchi A, Hamada H, Jing $\mathrm{N}$, Kondoh $\mathrm{H}$. Interplay of SOX and POU factors in regulation of the Nestin gene in neural primordial cells. Mol Cell Biol. 2004;24(20):8834-8846.

33. Krupkova O Jr, Loja T, Zambo I, Veselska R. Nestin expression in human tumors and tumor cell lines. Neoplasma. 2010;57(4):291-298.

34. Reh TA. Neurobiology: right timing for retina repair. Nature. 2006;444(7116):156-157.

35. Weber BN, et al. A critical role for TCF-1 in T-lineage specification and differentiation. Nature. 2011; 476(7358):63-68.

36. Van Raay TJ, et al. Frizzled 5 signaling governs the neural potential of progenitors in the developing Xenopus retina. Neuron. 2005;46(1):23-36.

37. Lamba D, Karl M, Reh T. Neural regeneration and cell replacement: a view from the eye. Cell Stem Cell. 2008;2(6):538-549.

38. Gamm DM, et al. Protection of visual functions by human neural progenitors in a rat model of retinal disease. PLoS One. 2007;2(3):e338.

39. Sauve Y, Pinilla I, Lund RD. Partial preservation of rod and cone ERG function following subretinal injection of ARPE-19 cells in RCS rats. Vision Res. 2006;46(8-9):1459-1472. 